\title{
Defective Anksla disrupts the export of receptor tyrosine kinases from the endoplasmic reticulum
}

\author{
Soochul Park ${ }^{*}$ \\ Department of Biological Science, Sookmyung Women's University, Seoul 04310, Korea
}

\begin{abstract}
EphA2 has been implicated in amplifying ErbB2 tumorigenic signaling. One protein that interacts with EphA2 is the Anks1a PTB adaptor. However, the precise role of Anks1a in EphA2mediated tumorigenesis is unclear. We demonstrated that Anks1a localizes to the ER upon phosphorylation and that the Ankyrin repeats and PTB of Anks1a bind to EphA2 and Sec23, respectively. Thus, Anks1a facilitates the selective packaging of EphA2 into COPII vesicles. Additionally, Anks1a knockout mice, a phenocopy of EphA2 knockout mice, exhibited markedly reduced ErbB2-induced breast tumorigenesis. Strikingly, ErbB2 did not localize to the cell surface following Anks1a knockdown in primary mammary tumor cells over-expressing ErbB2. Importantly, EphA2 was critical for stabilizing ErbB2 through complex formation, but its interaction with Anks1a also facilitated ErbB2 loading into COPII carriers. These findings suggest a novel role for Anks1a in the molecular pathogenesis of breast tumors and possibly other human diseases. [BMB Reports 2016; 49(12): 651-652]
\end{abstract}

Receptor tyrosine kinases (RTKs) play essential roles in the plasma membrane in terms of sensing and responding to dynamically changing environments. However, their translation, folding, and assembly must occur in the ER prior to being transported to the plasma membrane. Although COPII vesicle formation is a basic process underlying the export of proteins translated in the ER, few studies have analyzed the regulatory

*Corresponding author. E-mail: scpark@sookmyung.ac.kr

https://doi.org/10.5483/BMBRep.2016.49.12.186

Received 31 October 2016

Keywords: Anks1a, Breast tumor, ErbB2 (HER2, neu), PTB adaptor

Abbreviations: COPII, coat protein complex II; EGF, epidermal growth factor; ER, endoplasmic reticulum; ERES, ER exit site; PMTC, primary mammary tumor cell; PTB, phosphotyrosine binding; RTK, receptor tyrosine kinase

Perspective to: Haeryung Lee et al (2016), Anksla regulates COPII-mediated anterograde transport of receptor tyrosine kinases critical for tumorigenesis. Nature Communications, Sep 13;7:12799. doi: 10.1038/ncomms12799. mechanism facilitating the ER export of RTKs. The aberrant regulation of this process is an important cause of various human diseases, including cancer. Our recent report provides new insights into the functional role of PTB adaptor protein in the ER export of EphA2 and ErbB2 RTKs, which have critical roles in breast tumorigenesis.

ErbB2 is a member of the EGF receptor family, and its amplification or over-expression has been implicated in human breast cancer. EphA2 cooperates with ErbB2 to enhance breast tumor formation (Brantley-Sieders et al., J Clin Invest 2008, 118(1):64-78. doi:10.1172). Indeed, over-expression of EphA2 has been implicated in breast cancer and is associated with a poor prognosis. EphA2 associates in a complex with ErbB2 and stimulates the Ras-MAPK signaling pathway. EphA2 gene ablation impairs breast tumor formation in mice over-expressing ErbB2, providing a novel insight into therapies for breast cancer.

The Anks1 family of proteins consists of two members, Anks1a and Anks1b, and they contain six ankyrin repeats at the N-terminus, two SAM domains, and a PTB domain at the C-terminus. PTB adaptors are classified based on their structural differences, and Anks1 family adaptors belong to the Dab-like PTB adaptors. The Dab-like PTB adaptors play general roles in regulating transmembrane protein trafficking throughout the endomembrane system. Interestingly, the Anks1a adaptor has been shown to be downstream of the EGF and Eph receptor signaling pathways. However, it is unclear whether Anks1a is involved in the trafficking of RTKs in the ER or other organelles.

An initial key finding was that the cell surface level of EphA2 was markedly reduced in Anks1a-deficient MEFs. This phenotype could be explained by two potential mechanisms: either defective recycling of EphA2 or ineffective transport from the ER or Golgi. Importantly, a significant fraction of Anks1a was found to colocalize with ER markers, in particular, Sec16, a bona fide marker for the ER exit site (ERES). These two key findings led to the hypothesis that Anks1a adaptor interacts with EphA2 on the cytoplasmic side of the ER to regulate its anterograde transport. Indeed, it was demonstrated that the ankyrin repeats of Anks1a were critical for interacting with EphA2 in the ER. Importantly, the PTB domain of Anks1a was not involved in binding to EphA2 in the ER but was critical for facilitating the export of EphA2 from the ER. This 


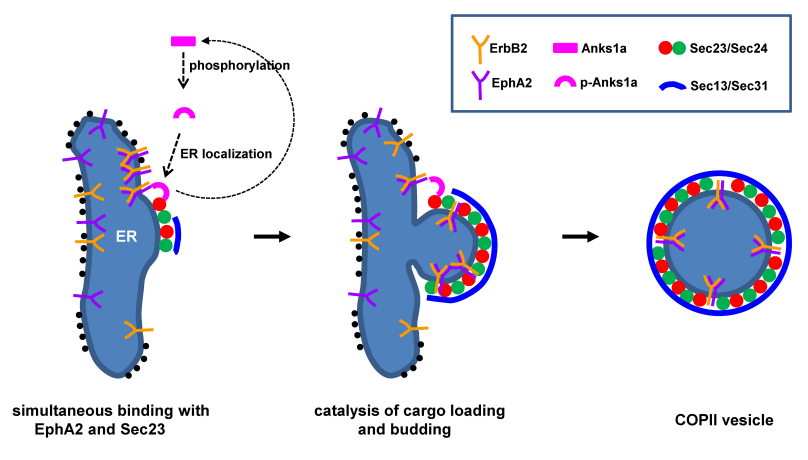

Fig. 1. Anks1a facilitates the export of EphA2/ErbB2 complex from the ER. In serum-stimulated cells, Ser-647 and -663 of human Anks1a are the major phosphorylation sites. Serine phosphorylation may change the protein structure of Anks1a so that phosphorylated Anks1a is localized to the ER. In the ER, Anks1a interacts simultaneously with EphA2 and Sec23 via two critical motifs: the Ankyrin repeats bind to EphA2 whereas the PTB domain binds to Sec23, a component of the COPII vesicle. Then, other COPII components (i.e., Sec24 and Sec13/31) are recruited to Sec23 in the ERES, deforming ER membrane for budding and possibly competing away Anks1a. This dynamic COPII biogenesis would catalyze the selective loading of some RTK cargos into the growing COPII vesicle.

key observation led to another important discovery that the PTB domain of Anks1a directly binds to Sec23, a component of COPII vesicles. Importantly, COPII vesicles play a pivotal role in the anterograde transport of cargo proteins from the ER to the Golgi.

It is unclear whether Anks1a plays a direct role in the selective loading of EphA2 into forming COPII vesicles. Results for the knock-down and gain-of-function of Anks1a appeared to be consistent with this predicted role. For example, an in vitro COPII vesicle budding assay using Anks1a knocked-down cells showed markedly reduced levels of EphA2 loaded into COPII vesicles, whereas the same assay using Anks1a over-expressing cells produced opposing results. However, when Anks1a was purified using a baculovirus expression system and then used in an in vitro vesicle budding assay, the result was unexpected, because Anks1a inhibited the loading of EphA2 into COPII vesicles. Additionally, the purified Anks1a protein was more effective in inhibiting rather than increasing the GAP activity of Sec23 toward Sar1-GTP, which is critical for the budding process of mature COPII vesicles in the ER. These contradictory results suggested that the purified Anks1a is somewhat different from the Anks1a protein acting in the ER of intact cells. Previous studies revealed that human Anks1a is a phosphoprotein that interacts with 14-3-3 protein and that Ser-647 and Ser-663 were major phosphorylation sites (Zhong et al., J Proteomics 2011, 74(3):294-303. doi: 10.1016). Importantly, a phosphorylationdefective Anks1a mutant neither localized to the ER nor stimulated the loading of EphA2 into COPII vesicles. These results support the hypothesis that the post-translationally modified form of Anks1a plays a role in the selective packaging of EphA2 into forming vesicles in the ERES (Fig. 1).

An important issue is how Anks1a influences breast tumorigenesis. It was predicted that the ineffective ER export of EphA2 in the absence of Anks1a would decrease the oncogenic signaling of ErbB2 in a mouse breast tumor model. The results in Anks1a knockout mice consistently showed that breast tumorigenic potential was markedly reduced, despite overexpression of ErbB2 under the MMTV promoter. Strikingly, primary mammary tumor cells from MMTV-Neu mice displayed very low levels of both EphA2 and ErbB2 on the cell surface when Anks1a was knocked down. It was further shown that EphA2 is critical for stabilizing ErbB2 and that they form a complex in the ER. In addition, Anks1a was shown to stimulate the selective loading of the EphA2/ErbB2 complex into the forming COPII vesicles. This novel finding provides strong evidence that oncogenic signaling of ErbB2 on the cell surface requires two distinct features of EphA2: protein stabilization through the formation of a large receptor complex and more efficient loading into COPII vesicles.

Many questions remain, such as whether the specific binding of the PTB domain to Sec23 is conserved among other PTB adaptors, in particular, Dab-like PTB adaptor proteins. Another important question is whether Anks1a expression is aberrantly regulated in breast cancer patients. Overall, our study represents a critical step forward towards understanding the molecular pathogenesis of breast tumors and other human diseases.

\section{ACKNOWLEDGEMENTS}

This work was supported by grants 2013M3C7A1056565 and 2015R1A2A1A15052871 from the National Research Foundation of Korea (NRF). 